\title{
Chronic antihypertensive therapy with thiazide diuretics in older women and risk of osteoporotic vertebral fractures: A recently much-debated association
}

Running title: Thiazide-related hyponatremia and osteoporotic vertebral fractures

Renato De Vecchis' ${ }^{1}$ Carmelina Ariano ${ }^{1,2}$, Arturo Cesaro ${ }^{3}$, Giuseppina Di Biase ${ }^{4}$

1 Cardiology Unit, Presidio Sanitario Intermedio "Elena d'Aosta", ASL Napoli 1 Centro, via Cagnazzi 29, 80137 Napoli, Italy. e-mail address: devecchis.erre@virgilio.it

2 Division of Cardiology, Casa di Cura "Sollievo della Sofferenza", viale Cappuccini 2, 71013 San Giovanni Rotondo, Italy. e-mail address: carmelariano@tiscali.it

3 Department of Cardiology, Second University of Napoli, Monaldi Hospital, via Leonardo Bianchi 1, 80131

Napoli, Italy. e-mail address: carmelina.ariano.e7gn@na.omceo.it

4 Division of Geriatrics, Neurorehabilitation Unit, Clinic "S. Maria del Pozzo", via Pomigliano 40, 80049

Somma Vesuviana, Italy. e-mail address: smariaves268@gmail.com

Corresponding author: Dr Renato De Vecchis, MD, FESC

Cardiology Unit, Presidio Sanitario Intermedio "Elena d'Aosta", ASL Napoli 1 Centro, via Cagnazzi 29, 80137

Napoli, Italy; devecchis.erre@virgilio.it.

\section{Abstract}

Background An alleged association of chronic use of thiazide diuretics with an increased risk of bone fragility fractures has been highlighted by a relatively recent prospective cohort study (Am J Med. 2016 Dec; 129(12):1299-1306). However, the concept that thiazides exert a beneficial effect on osteoporosis is still a predominant view. This effect would be mediated by the decrease in renal clearance of calcium ions, a pharmacological feature recognized for a long time now to this class of drugs, as opposed to the increase in calcium urinary excretion attributed instead to loop diuretics, i.e. furosemide and similar drugs. The purpose of this retrospective study is to attempt to clarify whether regular use of thiazide diuretics as antihypertensive therapeutics is associated with a significantly increased risk of osteoporotic fractures in female patients, aged over 70 years.

Methods In this two-centre retrospective study, we followed up a cohort of female patients with $(n=80)$ and without $(n=158)$ thiazide-induced hyponatraemia.

Results A total of 48 osteoporotic fractures was recorded during a median followup period of 57.5 months. By means of univariate regression analysis, an association was found between thiazide-induced hyponatraemia and increased risk of vertebral fractures (Odds ratio [OR]: 7.6; 95\% confidence interval [CI]: 3.755 - 15.39; $\mathrm{p}<0.0001)$. Multivariate regression analysis, however, showed that age (OR: 1.823; 95\% CI: 1.211 to 2.743 ) and body mass index (OR: 0.156 ; 95\% CI: 0.038 to 0.645) were the only independent predictors of osteoporotic fractures. No association of a history of thiazide-induced hyponatraemia and risk of fracture was noticeable in the final model. 
Conclusions Because a history of thiazide-induced hyponatremia is associated with osteoporotic fracture in univariate but not multivariate analyses, a possible explanation is that confounding factors of older age and low body mass index are responsible for the apparently heightened risk of fragility fractures in hypertensive female patients with thiazide-related hyponatremia.

Keywords: thiazide diuretics; cardiovascular risk; osteoporosis; vertebral fractures

\section{Manuscript}

\section{Background}

There is a fair amount of studies that have dealt with exploring the possible relationship between the use of diuretics (both thiazides and loop diuretics) and development of osteoporotic fractures (1-8).

Among the most recent studies, there is an observational study by Paik JM et al (9). In this prospective cohort study of thiazide diuretic use, loop diuretic use and risk of incident clinical vertebral fracture, Cox proportional-hazards regression analysis documented a significantly increased risk of developing vertebral fractures in postmenopausal women taking diuretics. Indeed, thiazide use was associated with an increased risk of clinical vertebral fracture $(R R=1.47$; 95\% CI: 1.18-1.85). Similarly, women receiving a loop diuretic were more likely to be affected by clinical vertebral fracture ( $R R=1.59 ; 95 \% C I: 1.12-2.25)$ compared to women not treated with a loop diuretic. The higher risk of vertebral fracture with thiazide use was unexpected.

In fact, despite the above-mentioned recent study, a thiazide-induced osteoporotic effect is currently not recognized by the vast majority of studies (1014).

Moreover, in order to distinguish between the osteo-toxic effect of loop diuretics and that presumably attributed to the thiazide diuretics, it should be noted that the possible mechanisms for loop diuretics include their calciuric effect, subsequent increase in plasma parathyroid hormone level, and decreased bone mineral density (15-17). Instead, the alleged harmful effect on the vertebral bone arising from the chronic use of thiazides would be mediated by their strong ability to deplete the body's sodium pool.

Skeletal bone is a rich reservoir of sodium (18) and may play a key role in maintaining sodium homeostasis (19) but possibly at the expense of the bone's structural integrity. The spine is particularly vulnerable to micro-damage ,e.g., linear microcracks and diffuse damage, (20) compared with other fracture sites, considering its dissimilar biomechanics as well as different loading indexes, microarchitecture, constitution, geometry, and structural arrangement (21 -23), and the microdamage accumulates with aging(24). The increased vertebral fracture risk 
seen with thiazide use may be induced by the potential harmful effect of hyponatremia on the spine's ability to repair local micro-damage.

Thus, a retrospective study has been carried out to clarify whether, in our experience, the regular use of thiazide diuretics administered as anti-hypertensive therapy may be associated with a significantly increased risk of osteoporotic vertebral fractures in female patients, older than 70 years.

\section{Methods}

In this two-centre retrospective study, we followed up a cohort of female patients with and without thiazide-induced hyponatraemia. The relevant data were collected by review of medical records that were thoroughly analyzed .In particular, in order to be admitted to the present case-control study, patients had to be female older than 70 years at the time of their first visit, they had to have undergone one or more hospitalizations or specialist visits to one of the two hospitals involved in the study (C S d $\mathrm{S}$ and $\mathrm{S} . \mathrm{M} \mathrm{d} \mathrm{P)} \mathrm{and} \mathrm{had} \mathrm{to} \mathrm{have} \mathrm{received} \mathrm{the} \mathrm{diagnosis} \mathrm{of} \mathrm{hypertension.} \mathrm{In} \mathrm{addition,} \mathrm{a}$ further requirement for enrollment in this retrospective study was that antihypertensive therapy should consist of a thiazide diuretic (hydrochlorothiazide, chlortalidone, indapamide, metolazone, etc.) taken alone, or in combination with another antihypertensive agent not belonging to the class of thiazide diuretics, on a daily basis for a period not less than 6 months.

Particular attention was paid to the finding of any hyponatremia (isolated or repeated) in the patient history. Hyponatraemia was defined by a serum sodium level below $135 \mathrm{mEq} /$ 1. Moreover, special attention was paid to the diagnostic evaluation of cases of osteoporotic vertebral fracture. The study included a scrupulous anamnestic investigation able to highlight a possible history of fracture, lesion, or collapse of one or more vertebrae. In the vast majority of cases, all relevant information was acquired without directly contacting patients, but simply through the scrupulous examination of the history and clinical data contained in the medical records. Data was then collected, processed and analyzed for statistical assessment at a third Structure (E.d' A.).

For the consultation of clinical documentation, attention was paid to the careful preservation of patients' privacy and compliance with current regulations that recommend collecting clinical record data in anonymous aggregate form for conducting case-control retrospective studies. The Hospital Directorates were informed in this regard,while no informed consent of the patients nor the preventive interpellation of the Ethics Committee was deemed necessary, since it was a non-experimental retrospective study conducted, while the due attention was paid to the anonymity of the patient. The group "Patients with history of one or more vertebral fractures"included those who had, in the medical record, the documentation of their osteo-vertebral pathology in the form of a radiological report (e.g., radiograph, computerized tomography scan or magnetic resonance imaging). However, even a simple medical report (e.g, clinic visit notes, operative 
notes, hospital discharge summary) was considered sufficient to diagnose a previous osteoporotic fracture of the spine.

We included vertebral fractures that were related to low or moderate trauma (eg, tripping, or falling from the height of a chair or lower). We excluded vertebral fractures due to high trauma (e.g., fall down a flight of stairs), motor vehicle accidents, bicycle accidents, or horseback riding accidents. We also excluded cases of cervical or sacral fracture. We included in the analysis only cases of vertebral fracture that were confirmed by medical record review and diagnosed during the 6 years of follow-up between 2011 and May 31, 2017. Participants with a history of hip or wrist fracture at baseline were excluded from the analysis.

\section{Statistical analysis}

Patient data, including smoking status, diabetes mellitus, body mass index, walking ability, status of living in geriatric longstay facility or nursing homes (vs home living) and other demographic characteristics, were collected.

Data were expressed in mean \pm SD unless otherwise specified. For single comparisons, ANOVA- one way method was used. When judged useful, Cstatistics for building receiving operator characteristic(ROC) curve was also used, in order to ascertain the optimal cut-off provided with the best balance between sensitivity and specificity, in the case of quantitative exposure variables that had been recognized to exert a role as predictive factors for vertebral fractures in hypertensive elderly women treated with thiazide diuretics. A subdivision of the enrolled patients according to their serum $\mathrm{Na}^{+}$levels was also performed. Based on this categorization, three serum sodium classes were identified i.e., class 0 , consisting of normonatremic patients, class 1 , consisting of patients with serum sodium level of 115-134 mEq / 1, and class 2, consisting of patients with serum sodium level $<115 \mathrm{mEq} / 1$. This distinction was made to ascertain whether higher severity of hyponatremia was associated with a proportionately greater risk of newly diagnosed osteoporotic vertebral fractures. We then examined the association of thiazide- induced hyponatraemia with fracture occurrence using 2 per 2 contingency tables and multivariate logistic regression analysis. The exposure variables entered in the model were age, body mass index, thiazideinduced hyponatremia, smoking status, walking ability, status of living in geriatric longstay facility and diabetes mellitus. A history of thiazide-induced hyponatremia, age, body mass index and the variables at an alpha level $<0.1$ in univariate analysis were selected for multivariate analysis. Two-tailed $p$-values < 0.05 were considered statistically significant. Statistical analyses were performed using SPSS software 16.0 (SPSS, Chicago, IL, USA). 


\section{Results}

Baseline clinical and demographic data are shown in Table 1. The group of elderly hypertensive female patients with thiazide-induced hyponatraemia was composed of 80 individual whereas the one without thiazide-induced hyponatraemia included 158 subjects. The mean age of the 80 subjects with thiazide-induced hyponatremia was $74 \pm 8.5$ years, significantly older than the 158 subjects without thiazide-induced hyponatremia (age $65 \pm 7.5$ years; $p<0.0001$ ) (Figure 1)

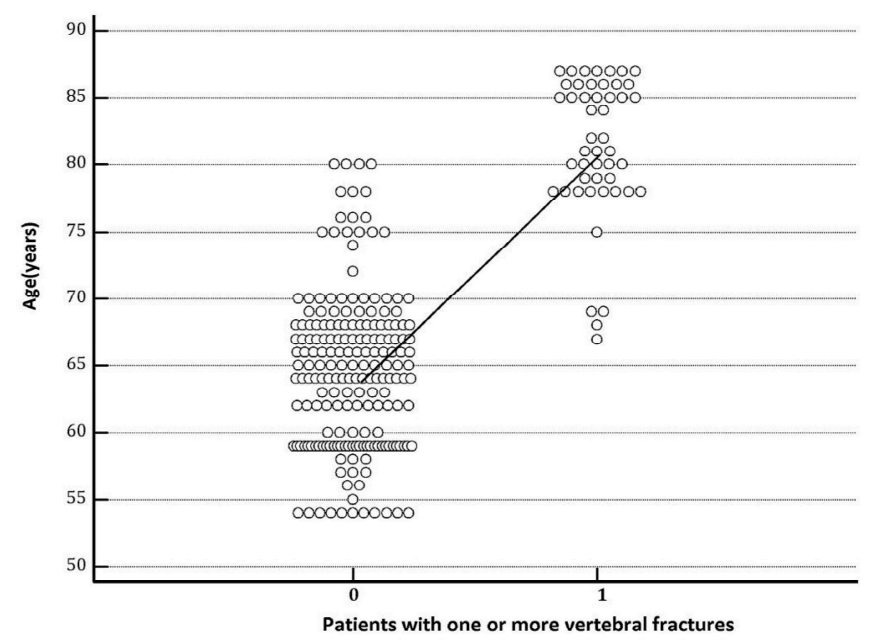

\footnotetext{
Fig 1 The column on the right represents the group of hypertensive patients who experienced one or more vertebral fractures during the follow-up. The graph clearly shows that mean age recorded at the entrance into the study was significantly higher $(\mathbf{p}<0.001)$ in hypertensive patients with destructive osteoporosis of the spine, i.e., which developed one or more vertebral fractures ( age: $81.33 \pm 5.27$ years) compared with those that did not have vertebral collapses (age: $64.53 \pm 5$. 86 years) ; see also the prospectus at the bottom of the figure.

Descriptive Statistics for Each Value of Crosstab Variable

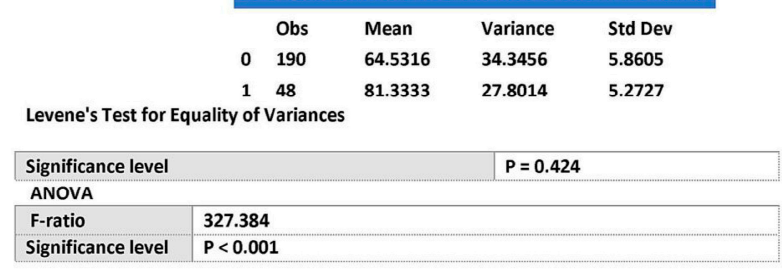

Student-Newman-Keuls test for all pairwise comparisons

\begin{tabular}{|l|l|l|l|}
\hline Factor & $\mathrm{n}$ & Mean & $\begin{array}{l}\text { Different }(\mathrm{P}<0.05) \\
\text { from factor } \mathrm{nr}\end{array}$ \\
\hline $\mathbf{1 0}$ & 190 & 64.5316 & $(\mathbf{1})$ \\
\hline$(1)$ & 48 & 81.3333 & $(0)$ \\
\hline
\end{tabular}
}


Table 1 Baseline demographic characteristics

\begin{tabular}{|l|c|c|l|}
\hline \multicolumn{5}{|l|}{} \\
\hline & $\begin{array}{l}\text { Patients with thiazide-induced } \\
\text { hyponatremia( no. 80) }\end{array}$ & $\begin{array}{l}\text { Patients without hyponatremia } \\
\text { on thiazide (no.158) }\end{array}$ & $p$-value \\
\hline Age ( years) & $74 \pm 8.5$ & $65 \pm 7.5$ & $p<0.0001$ \\
\hline Body mass index $\left(\mathrm{kg} / \mathrm{m}^{2}\right)$ & $22.6 \pm 4.17$ & $24.19 \pm 3.38$ & $p=0.0017$ \\
\hline Duration of follow-up(months) & $56.1 \pm 10.5$ & $54.8 \pm 10.5$ & $p=0.3705$ \\
\hline Ever smoked & $23(28.7 \%)$ & $48(30.3 \%)$ & $P=0.91$ \\
\hline Independent walking ability & $50(62.5 \%)$ & $128(81 \%)$ & $p=0.0032$ \\
\hline Diabetes mellitus & $5(6.25 \%)$ & $3(2 \%)$ & $p=0.122$ \\
\hline $\begin{array}{l}\text { Living in long-stay geriatric } \\
\text { facility }\end{array}$ & $3(4 \%)$ & $11(7 \%)$ & $p=0.394$ \\
\hline
\end{tabular}

Legend Continuous variables are expressed as mean \pm standard deviation

As previously reported, the hyponatremia group was also of lower body mass index $\left(22.6 \pm 4.17 \mathrm{~kg} / \mathrm{m}^{2}\right.$ versus $\left.24.19 \pm 3.38 \mathrm{~kg} / \mathrm{m}^{2} ; p=0.0017\right)$ (Figure 2$)$. In addition, in Figure 3, a ROC-plot highlights the predictive value for occurrence of osteoporotic vertebral fractures exhibited by body mass index.

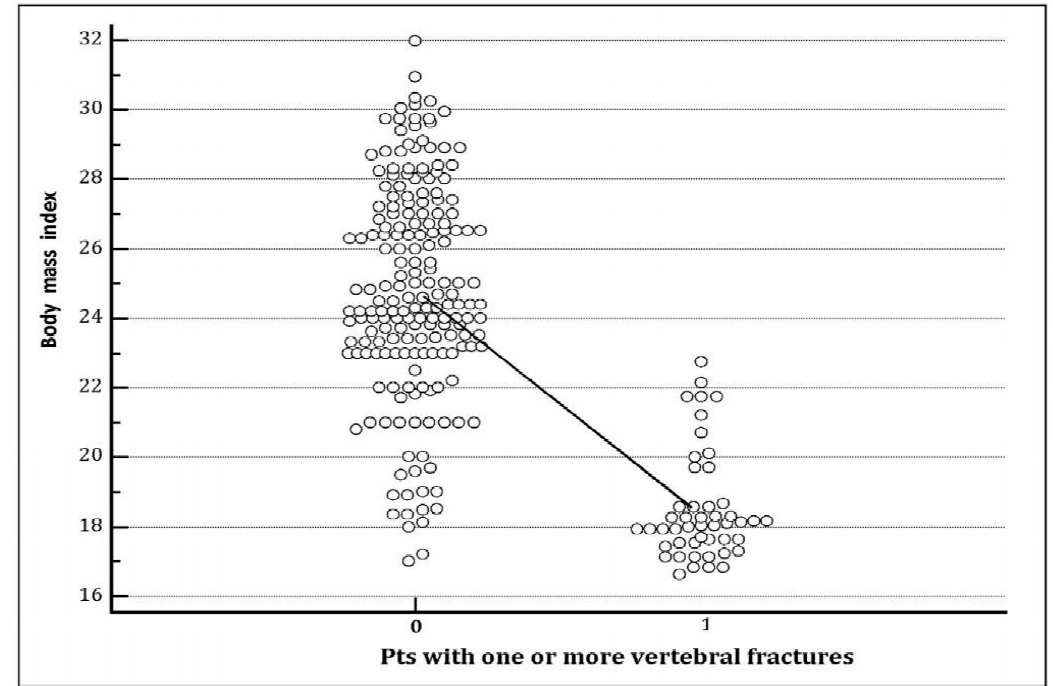

Fig.2 The column on the right represents the group of hypertensive patients who experienced one or more vertebral fractures during the follow-up. The chart clearly shows that mean body mass index (BMI) recorded at the entrance into the study was significantly lower $(p<0.001)$ in hypertensive patients with destructive osteoporosis of the spine, i.e., which developed one or more vertebral fractures ( BMI: $18.85 \pm 1.52$ ) compared with those that did not have vertebral collapses (BMI: 24.86 $\pm 3.10)$; see also the prospectus at the bottom of the figure)

\section{Descriptive Statistics for Each Value of Crosstab Variable}

$\begin{array}{ccccc} & \text { Obs } & \text { Mean } & \text { Variance } & \text { Std Dev } \\ 0 & 190 & 24.8636 & 9.6550 & 3.1072 \\ 1 & 48 & 18.8577 & 2.125 & 1.5207\end{array}$

Levene's Test for Equality of Variances

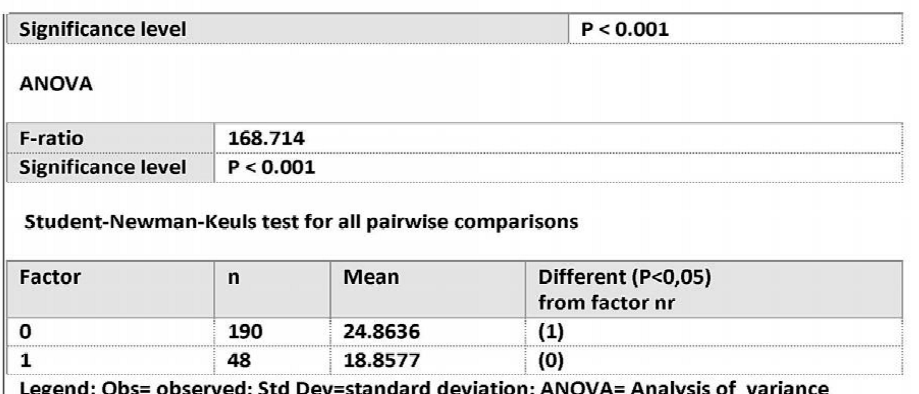

Mean serum sodium concentration was $123 \pm 9.5 \mathrm{mEq} / \mathrm{L}$ in the thiazideinduced hyponatremia group. Three cases developed seizures and four required intensive care unit admission. Mean hospitalisation duration was 6 days for the hyponatremia group. 


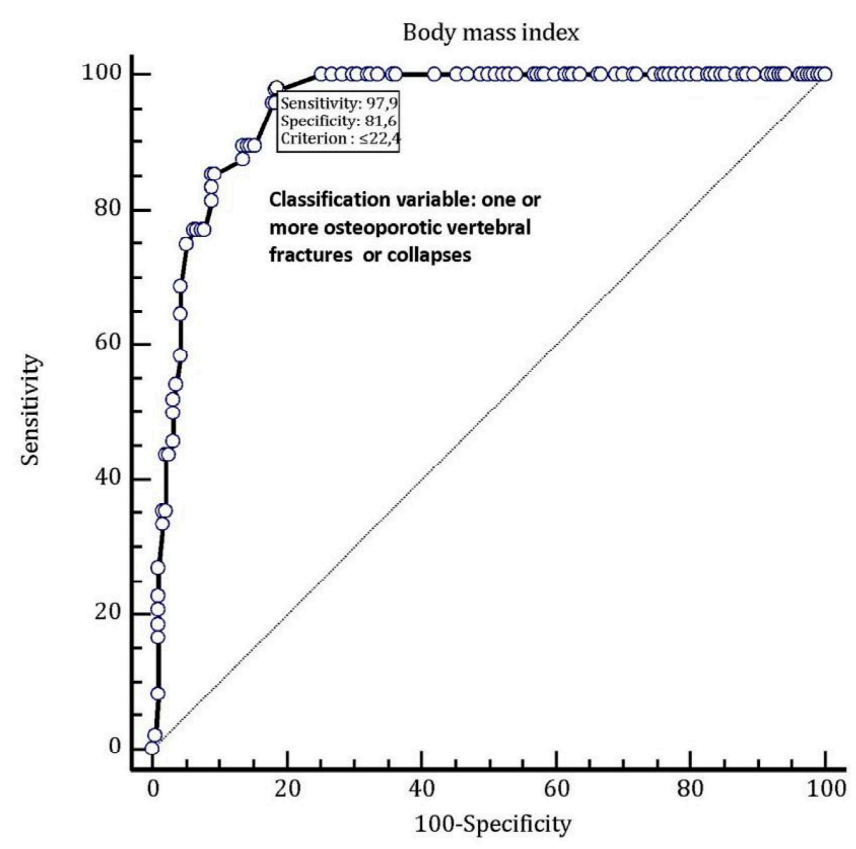

Sample size

Area under the ROC curve (AUC)

\begin{tabular}{|l|r|}
\hline Area under the ROC curve (AUC) & 0,950 \\
\hline Standard Error $^{\mathrm{a}}$ & 0,0132 \\
\hline $95 \%$ Confidence interval & ${ }^{\mathrm{b}}$ \\
\hline z statistic & 0,914 to 0,974 \\
\hline Significance level P (Area $=0.5$ ) & 34,042 \\
\hline
\end{tabular}

Fig.3

In this ROC plot, there is the representation of the very good predictive value ( $A U C=0.950$ ) possessed

by body mass index ( $\mathrm{BMI}$ ) as a tool for predicting the occurrence of one or more osteoporotic

vertebral fractures in hypertensive female patients older than 70 years taking chronic therapy with

an oral thiazide diuretic (alone or in combination with another anti-hypertensive drug). By adopting

this method, the best diagnostic accuracy for predicting an osteoporotic spinal fracture has been

attributed to the BMI threshold value of 22.4. This means that in the enrolled population of thiazide

users this value is associated to the presence of osteoporotic vertebral collapse or fracture with a

sensitivity of $97.9 \%$ and a specificity of $\mathbf{8 1 . 6 \%}$ (note on top of the graph).

Median follow up was of 57.5 months (interquartile range $54-60$ ) in the entire cohort of enrolled patients.

Furthermore 34 individuals (42.5\%) with thiazide induced hyponatremia and $14(8.8 \%)$ without thiazide-induced hyponatremia experienced osteoporotic vertebral fractures (Table 2) (odds ratio: 7.60; 95\% confidence interval [CI] : 3.75515.39; p [ chi - square test with Yate correction] <0.0001). Thus, thiazide-induced hyponatremia was an univariate predictor associated with an increased risk of newly diagnosed, osteoporotic vertebral fractures. 


\begin{tabular}{|c|c|c|c|c|}
\hline \multicolumn{2}{|l|}{} & \multicolumn{3}{|c|}{ Patients with one or more vertebral fractures } \\
\hline & & yes & no & Total \\
\hline \multirow{2}{*}{} & yes & 34 & 46 & 80 \\
\hline \multirow{2}{*}{} & & 14 & 144 & 158 \\
\cline { 2 - 5 } & no & 48 & & 238 \\
\hline & Total & & 190 & \\
\hline
\end{tabular}

\begin{tabular}{|c|c|}
\hline Odds ratio & 7.6025 \\
\hline $95 \% \mathrm{Cl}$ & 3.755 to 15.3923 \\
\hline 2 statistic & 5.636 \\
\hline significance level & $\mathrm{P}<0.0001$ \\
\hline
\end{tabular}

Table 2 This $2 \times 2$ contingency table shows that, in female hypertensive patients older than 70 years taking thiazide diuretics, the odds of experiencing one or more osteoporotic vertebral fractures is significantly $(p<0.0001)$ higher among patients with hyponatremia( serum sodium level $<135 \mathrm{mEq} / \mathrm{l}$ ) compared to those with normal serum sodium levels (odds ratio:7.6025; 95\% $\mathrm{Cl}: 3.755$ to 15.3923$)$. For further explanations, please see the text.

Legend: $\mathrm{Cl}=$ Confidence interval

Table 3 shows the results of multivariate logistic regression analysis for the predictors of osteoporotic fractures. In this way, using multivariate logistic regression to estimate the probability of any osteoporotic vertebral fracture, we found that thiazide-induced hyponatremia was not any longer significantly associated with fracture risk. Instead, the only two multivariate predictors of vertebral fractures were patient age (odds ratio(OR) $=1.823 ; 95 \%$ CI: 1.211 to 2.743 ; $p=0.0039)$ and body mass index ( $\mathrm{OR}=0.156 ; 95 \%$ CI:0.038 to $0.645 ; p=0.0102)$.In addition, the two classes of hyponatremia we arbitrarily created had a comparable odds of incident vertebral fractures, that is, newly diagnosed vertebral fractures were similarly frequent in the comparison between the two classes, namely the one with serum $\mathrm{Na}^{+}$of $<115 \mathrm{mEq} / \mathrm{L}$, compared to that whose values were only moderately depressed, i.e., comprised between 115 and $134 \mathrm{mEq} / 1$. 
TABLE 3 Multivariate logistic regression analysis outcome variable: patients with one or more vertebral fractures exposure variables: age( continuous);body mass index ( continuous); hyponatremia during thiazide therapy( dichotomous); inability for independent walking( dichotomous); living in geriatric long stay facility( dichotomous); diabetes (dichotomous)

Odds Ratios and 95\% Confidence Intervals

\begin{tabular}{|l|c|c|c|c|c|}
\hline Variable & Coefficient & Std. Error & Odds ratio & $95 \% \mathrm{Cl}$ & $\boldsymbol{p}$ \\
\hline Age ( continuous variable) & 0.600 & 0.208 & 1.823 & 1.211 to 2.743 & 0.0039 \\
\hline $\begin{array}{l}\text { Body mass index( continuous } \\
\text { variable) }\end{array}$ & -1.852 & 0.721 & 0.156 & 0.038 to 0.645 & 0.0102 \\
\hline $\begin{array}{l}\text { Hyponatremia under thiazide } \\
\text { treatment }\end{array}$ & 2.970 & 1.681 & 19.508 & 0.722 to 527.094 & 0.0773 \\
\hline $\begin{array}{l}\text { Inability for independent } \\
\text { walking }\end{array}$ & -3.318 & 2.289 & 0.036 & 0.0004 to 3.216 & 0.1472 \\
\hline $\begin{array}{l}\text { Living in geriatric long stay } \\
\text { facility }\end{array}$ & -3.136 & 2.482 & 0.043 & 0.0003 to 5.630 & 0.2063 \\
\hline \begin{tabular}{l} 
Diabetes \\
\hline
\end{tabular} & 2.217 & 1.556 & 9.179 & 0.434 to 193.958 & 0.1543 \\
\hline
\end{tabular}

Table 3 Multivariate predictors of occurrence of osteoporotic vertebral fractures ( one or more for each individual) among hypertensive female patients older than 70 years, all taking chronic anti-hypertensive therapy with thiazide diuretic ( alone or in combination with other anti-hypertensive agents). As reported in the table, hyponatremia per se is not associated with significantly increased risk of osteoporotic vertebral fractures $(p=0.0773)$. On the contrary, advanced age appears to be a risk factor $(p=0.0039)$, whereas a relatively high body mass index appears to exert a significant protective effect (OR: 0.156; $95 \% \mathrm{Cl}$ : 0.038 to $0.645 ; p=0.0102$ ) against occurrence of osteoporotic vertebral fractures.

Legend: Cl: confidence interval; Std. Error: standard error

\section{Discussion}

Use of diuretics for treatment of hypertension, as well as chronic heart failure has been associated with a reduction in bone mineral density, and related increased risk of osteoporotic fractures, especially at the vertebral level (25-26). This topic has attracted the interest of epidemiologists and clinicians, also because the propitiation or aggravation of osteoporosis may substantially cancel or diminish the overall therapeutic advantage arising from the beneficial effect on the cardiovascular apparatus exerted by thiazide diuretics used for primary or secondary cardiovascular prevention( 27).

This unfavorable outcome would be due to the elicitation of osteoporosis in postmenopausal women as a harmful repercussion of the diuretics on scheletral structure.

There are controversial aspects regarding the supposed responsibility of diuretics in the determinism of osteoporosis. In principle, thiazide diuretics, i.e., those most commonly used in hypertension, alone or in combination with other pharmacological classes (ACE inhibitors, angiotensin receptor blockers, beta- 
blockers) are regarded not to usually increase, but rather decrease renal excretion of calcium ions( 28 ). Hence their participation in bone mineral depletion processes should be deemed to occur less likely when compared to that attributed to the loop diuretics (furosemide, torsemide, etacrinic acid).

In addition, relatively recently, low sodium serum levels have been blamed on causing a reduction in bone mineralization, especially in the elderly. In fact, thiazides, while not increasing calcium urinary excretion, may have an unfavorable role in bone mineral metabolism through their proved property of causing increase in sodium urinary clearance with possible related reduction in skeletal sodium stock (29). Moreover, while thiazide use has been found to be protective for hip fracture in several studies(10,13), other 2 studies of thiazide use and vertebral fracture (1-2) found no statistically significant association, whereas one (9)demonstrated significant association between thiazide use and incident clinical vertebral fractures.

As for the loop agents, also considered as the cornerstone of therapy for hemodynamic congestion in chronic heart failure, they have been shown to induce non-negligible hypercalciuria, thus making the bone more frail and friable and therefore more predisposed to pathological manifestations such as fragility fracture(16-17), namely the so-called vertebral collapse, defined by the crush of one or more vertebral bodies, often detectable in elderly with severe osteoporosis.

If epidemiological observations that would demonstrate the existence of a causal link between the use of diuretics and the occurrence of osteoporosis are confirmed by randomized trials with adequate statistical power, then therapeutic habits for treatment of hypertension and related prevention of cardiovascular and cerebrovascular events would be modified, i.e., a drastic reduction in the use of diuretics is likely to occur as a logic consequence from this finding.

In our retrospective case control study, although a history of thiazideinduced hyponatremia identified individuals at risk for osteoporotic fracture, the predictive value was no longer evident after controlling for known risk factors of osteoporosis. Indeed, only two

factors - namely, older age and lower body mass - maintained their predictive value with regard to osteoporotic vertebral fractures even in the multivariate logistic regression analysis.

Based on these findings, a significant association of osteoporotic vertebral fractures with older age and low body mass index can be affirmed. In other words, our findings indicate that old age and low body mass help explain the occurrence of osteoporotic vertebral fractures, and that hyponatraemia event might not be the culprit of the osteoporotic fractures.

\section{Conclusions}

At univariate logistic regression analysis, we found that a history of thiazideinduced hyponatremia in hypertensive women older than 70 years was associated with small increased risk of osteoporotic vertebral fracture. Conversely, in our 
study this association disappeared after adjustment for conventional risk factor of vertebral fractures, such as old age and low body mass index.

However, recent research has shown that use of diuretics (both thiazides and loop diuretics) is associated with an increased risk of vertebral fractures, namely the most typical manifestation of severe osteoporosis. Thus, a unifying thesis could be put forward as follows. Hyponatremia per se, doesn't induce osteoporotic fracture, however decreased bone mass (for instance, among elderly with small body build) might confer heightened risk of hyponatremia because of reduced bone sodium for exchange with the serum sodium. In keeping with this concept, occurrence of hyponatremia could only serve as an indirect surrogate marker for osteoporosis risk.

Conflict of interest statement On behalf of all authors, the corresponding author states that there is no conflict of interest.

\section{References}

1)Nevitt MC, Cummings SR, Stone KL, et al. Risk factors for a first incident radiographic vertebral fracture in women $>$ or $=65$ years of age: the study of osteoporotic fractures. J Bone Miner Res. 2005;20:131-140.

2) Rejnmark L, Vestergaard P, Mosekilde L. Reduced fracture risk in users of thiazide diuretics. Calcif Tissue Int. 2005;76:167-175.

3)Hoorn EJ, Rivadeneira F, van Meurs JB, et al. Mild hyponatremia as a risk factor for fractures: the Rotterdam Study. J Bone Miner Res. 2011;26:1822-1828.

4) Kinsella S, Moran S, Sullivan MO, Molloy MG, Eustace JA. Hyponatremia independent of osteoporosis is associated with fracture occurrence. Clin J Am Soc Nephrol. 2010;5:275-280.

5) Jamal SA, Arampatzis S, Harrison SL, et al. Hyponatremia and fractures: findings from the MrOS Study. J Bone Miner Res. 2015;30: 970-975.

6) Usala RL, Fernandez SJ, Mete M, et al. Hyponatremia is associated with increased osteoporosis and bone fractures in a large US health system population. J Clin Endocrinol Metab. 2015;100:3021-3031.

7) Sejling AS, Thorsteinsson AL, Pedersen-Bjergaard U, Eiken P. Recovery from SIADH-associated osteoporosis: a case report. J Clin Endocrinol Metab. 2014;99:3527-3530.

8) Upala S, Sanguankeo A. Association between hyponatremia, osteoporosis,

and fracture: a systematic review and meta-analysis. J Clin Endocrinol Metab. 2016;101:1880-1886.

9) Paik JM , Rosen HN , Gordon CM , Curhan GC . Diuretic Use and Risk of Vertebral Fracture in Women. Am J Med. 2016 Dec;129(12):1299-1306.

10)Lacroix AZ Thiazide diuretic agents and prevention of hip fracture. Compr Ther. 1991 Aug;17(8):30-9.

11)Cauley JA , Cummings SR, Seeley DG, Black D, Browner W, Kuller LH, Nevitt MC. Effects of thiazide diuretic therapy on bone mass, fractures, and falls. The Study of Osteoporotic Fractures Research Group.

Ann Intern Med. 1993 May 1;118(9):666-73.

12)Feskanich D, Willett WC, Stampfer MJ, Colditz GA. A prospective study of thiazide use and fractures in women. Osteoporos Int. 1997;7(1):79-84.

13)Schoofs MW, van der Klift M, Hofman A, de Laet CE, Herings RM, Stijnen T, Pols HA, Stricker BH. Thiazide diuretics and the risk for hip fracture. Ann Intern Med. 2003 Sep 16;139(6):476-82.

14)Aung $K$, Htay T. Thiazide diuretics and the risk of hip fracture. Cochrane Database Syst Rev. 2011 Oct 5;(10):CD005185. 
15) Friedman PA, Bushinsky DA. Diuretic effects on calcium metabolism.Semin Nephrol. 1999;19:551-556.

16) Rejnmark L, Vestergaard P, Heickendorff L, Andreasen F,Mosekilde L. Effects of long-term treatment with loop diuretics on bone mineral density, calcitropic hormones and bone turnover. J Intern

Med. 2005;257:176-184.

17) Rejnmark L, Vestergaard P, Heickendorff L, Andreasen F, Mosekilde L. Loop diuretics increase bone turnover and decrease BMD in osteopenic postmenopausal women: results from a randomized

controlled study with bumetanide. J Bone Miner Res. 2006;21: 163-170.

18)Bergstrom $\mathrm{WH}$, Wallace WM. Bone as a sodium and potassium reservoir. J Clin Invest. 1954;33:867-873.

19) Hannon MJ, Verbalis JG. Sodium homeostasis and bone. Curr Opin Nephrol Hypertens. 2014;23:370-376.

20) Seref-Ferlengez Z, Kennedy OD, Schaffler MB. Bone microdamage, remodeling and bone fragility: how much damage is too much damage? Bonekey Rep. 2015;4:644

21) Seeman E, Delmas PD. Bone quality - the material and structural basis of bone strength and fragility. N Engl J Med. 2006;354:2250-2261.

22) Bouxsein ML. Bone quality: where do we go from here? Osteoporos Int. 2003;14(suppl 5):S118S127.

23) Lambers FM, Bouman AR, Tkachenko EV, Keaveny TM,Hernandez CJ. The effects of tensilecompressive loading mode and microarchitecture on microdamage in human vertebral cancellous bone. J Biomech. 2014;47:3605-3612.

24) Arlot ME, Burt-Pichat B, Roux JP, Vashishth D, Bouxsein ML, Delmas PD. Microarchitecture influences microdamage accumulation in human vertebral trabecular bone. J Bone Miner Res. 2008;23:1613-1618.

25) Ayus JC , Bellido T, Negri AL. Hyponatremia and fractures: should hyponatremia be further studied as a potential biochemical risk factor to be included in FRAX algorithms? Osteoporos Int. 2017 May;28(5):1543-1548.

26) De Vecchis R, Noutsias M, Ariano C, Cesaro A, Cioppa C, Giasi A, Maurea N. Does Accidental Overcorrection of Symptomatic Hyponatremia in Chronic Heart Failure Require Specific Therapeutic Adjustments for Preventing Central Pontine Myelinolysis? J Clin Med Res. 2017 Apr;9(4):266-272.

27)Musini VM, Nazer M, Bassett K, Wright JM. Blood pressure-lowering efficacy of monotherapy with thiazide diuretics for primary hypertension. Cochrane Database Syst Rev. 2014 May 29;(5):CD003824.

28)Tsvetov G, Hirsch D, Shimon I, Benbassat C, Masri-Iraqi H, Gorshtein A, Herzberg D , Shochat T , Shraga-Slutzky I, Diker-Cohen T. Thiazide treatment in primary hyperparathyroidism-A new indication for an old medication? J Clin Endocrinol Metab. 2017 Apr 1;102(4):1270-1276

29)Hix JK, Silver S, Sterns RH. Diuretic-associated hyponatremia. Semin Nephrol. 2011 Nov;31(6):553-66. 